\title{
TRAJECTORIES UNDER A VECTORIAL POTENTIAL ON STATIONARY MANIFOLDS
}

\author{
ROSSELLA BARTOLO
}

Received 14 April 2001

\begin{abstract}
By using variational methods, we study the existence and multiplicity of trajectories under a vectorial potential on (standard) stationary Lorentzian manifolds possibly with boundary.
\end{abstract}

2000 Mathematics Subject Classification: 58E05, 58E10, 53C50.

1. Introduction and statement of the results. The pair $(\mathscr{L}, g)$ is called Lorentzian manifold if $\mathscr{L}$ is a connected finite-dimensional smooth manifold with $\operatorname{dim} \mathscr{L} \geq 2$ and $g$ is a Lorentzian metric on $\mathscr{L}$, that is, $g$ is a smooth symmetric two covariant tensor field such that for any $z \in \mathscr{L}$, the bilinear form $g(z)[\cdot, \cdot]$ induced on $T_{z} \mathscr{L}$ is nondegenerate and of index $v(g)=1$. Its points are called events. A Lorentzian manifold $(\mathscr{L}, g)$ is called (standard) stationary if $\mathscr{L}$ is a product manifold

$$
\mathscr{L}=\mathcal{M} \times \mathbb{R}, \quad \mathcal{M} \text { any connected manifold }
$$

and $g$ can be written as

$$
\left\langle\zeta, \zeta^{\prime}\right\rangle_{L}=\left\langle\xi, \xi^{\prime}\right\rangle+\langle\delta(x), \xi\rangle \tau^{\prime}+\left\langle\delta(x), \xi^{\prime}\right\rangle \tau-\beta(x) \tau \tau^{\prime}
$$

for any $z=(x, t) \in \mathscr{L}, \zeta=(\xi, \tau), \zeta^{\prime}=\left(\xi^{\prime}, \tau^{\prime}\right) \in T_{z} \mathscr{L}=T_{x} \mathcal{M} \times \mathbb{R}$, where $\langle\cdot, \cdot\rangle$, $\delta$, and $\beta$ are, respectively, a Riemannian metric on $\mathcal{M}$, a smooth vector field, and a smooth scalar field on $\mathcal{M}$. We refer to $[13,15,17]$ for all the background material assumed in this paper. Let $A$ be a smooth stationary vector field on $\mathscr{L}$, that is,

$$
A(z)=A(x, t)=A(x)=\left(A_{1}(x), A_{2}(x)\right) \quad \forall z=(x, t) \in \mathscr{L},
$$

and let $\mathscr{F}$ be the $(1,1)$ tensor field associated to $\operatorname{curl} A$. In this paper, we look for smooth curves $\gamma:[0,1] \rightarrow \mathscr{Y}$ (trajectories) which solve the problem

$$
\begin{gathered}
D_{s} \dot{\gamma}(s)=\frac{1}{2} \mathscr{F}(\gamma(s))[\dot{\gamma}(s)], \\
\gamma(0)=z, \quad \gamma(1)=w,
\end{gathered}
$$


where $z$ and $w$ are two fixed events of $\mathscr{Y}, \mathscr{Y}$ is an open connected subset of $\mathscr{L}$. When $\gamma$ is a trajectory, there exists $E_{\gamma} \in \mathbb{R}$ such that

$$
\langle\dot{\gamma}(s), \dot{\gamma}(s)\rangle_{L}=E_{\gamma} \quad \forall s \in[0,1]
$$

(see Remark 2.2) thus its causal character is well defined. We point out that (1.4) represents the Lorentz world-force law which determinates the motion of relativistic particles submitted to an electromagnetic field, when we take into account timelike curves $\gamma$ (see [17, page 88]). In this case, $A_{1}$ is called vectorial potential and $A_{2}$ is called scalar potential, see [12]. It is clear that this problem generalizes the one of the geodesic connectedness (see, e.g., [6, 11, 13]). Our problem has a variational nature. Indeed trajectories connecting two events are the critical points of the functional

$$
F(\gamma)=\int_{0}^{1}\langle\dot{\gamma}, \dot{\gamma}\rangle_{L} d s+\int_{0}^{1}\langle A(\gamma), \dot{\gamma}\rangle_{L} d s
$$

on a suitable infinite-dimensional manifold, see [5] and Section 2. When the manifold is (standard) static, existence and multiplicity results for these trajectories have been found in [2] and very recently timelike trajectories on stationary complete manifolds have been studied in [9]. For results on periodic trajectories, we refer to $[4,9,14]$.

In the following, for any vector $\xi \in T \mu$, we set $|\xi|=\sqrt{\langle\xi, \xi\rangle}$. Now, we are ready to state our first result, where we assume the completeness of $\mathscr{L}$.

THEOREM 1.1. Let $\left(\mathscr{L},\langle\cdot, \cdot\rangle_{L}\right)$ be a stationary Lorentzian manifold with $M$ complete and assume that

(i) there exist $\eta, b, d \in \mathbb{R}$ such that

$$
\begin{gathered}
0<\eta \leq \beta(x) \leq b \quad \forall x \in \mathcal{M}, \\
\sup _{x \in \mathcal{M}}|\delta(x)|=d ;
\end{gathered}
$$

(ii) there exist $a_{1}, a_{2} \in \mathbb{R}$ such that

$$
\sup _{x \in \mathcal{M}}\left|A_{1}(x)\right|=a_{1}, \quad 0 \leq A_{2}(x) \leq a_{2} .
$$

Then, for each two given events in $\mathscr{L}$ a trajectory y joining them exists. Moreover, if $\mathcal{M}$ is noncontractible in itself, then, for each two given events of $\mathscr{L}$ a sequence $\left\{\gamma_{m}\right\}$ of trajectories joining them exists.

REMARK 1.2. A gauge transformation does not modify (1.4). Indeed adding to $A$ any irrotational vector field $B$ independent on $t$, say $B(x, t)=\left(\nabla V(x), a_{0}\right)$ with $V \in \mathscr{C}^{2}(\mathcal{M}, \mathbb{R})$ and $a_{0} \in \mathbb{R}$, the critical points of the corresponding functional satisfy the same Euler-Lagrange equation. Thus it is enough that $A+B$ satisfies assumption (ii) of Theorem 1.1 for such $B$ (in particular it suffices that $A_{2}$ is bounded from below). 
We also deal with noncomplete stationary Lorentzian manifolds having boundaries satisfying some convexity assumptions. Let $\mathscr{S}$ be an open domain of a Lorentzian manifold $\mathscr{L}, \partial \mathscr{Y}$ its differentiable topological boundary and $\overline{\mathscr{S}}=$ $\mathscr{T} \cup \partial \mathscr{S}$.

We recall the following definition.

DEFINITION 1.3 (global convexity, variational point of view). We say that $\partial \mathscr{S}$ is convex if and only if for one, and then for all, nonnegative function $\Phi$ on $\overline{\mathscr{S}}$ such that

$$
\begin{gathered}
\Phi^{-1}(0)=\partial \mathscr{S}, \\
\Phi>0 \quad \text { on } \mathscr{Y}, \\
\nabla^{L} \Phi(z) \neq 0 \quad \forall z \in \partial \mathscr{},
\end{gathered}
$$

it results that

$$
H_{\Phi}^{L}(z)[\zeta, \zeta] \leq 0 \quad \forall z \in \partial \mathscr{S}, \zeta \in T_{z} \partial \mathscr{S}
$$

In [3], it has been proved that the previous definition is equivalent to the following one.

DEFINITION 1.4 (global convexity, geometrical point of view). We say that $\partial \mathscr{Y}$ is convex if for any $z, w \in \mathscr{Y}$ the range of any geodesic $\gamma:[0,1] \rightarrow \overline{\mathscr{S}}$ such that $\gamma(0)=z, \gamma(1)=w$ satisfies

$$
\gamma([0,1]) \subset \mathscr{S} .
$$

We recall that also the definition of causal convexity can be given, see, for example, [7] (see also [3]).

We use the following definition.

DEFINITION 1.5. A manifold $\left(\mathscr{Y},\langle\cdot, \cdot\rangle_{L}\right)$, with $\mathscr{Y}=\mathscr{D} \times \mathbb{R}$, is said to be a stationary Lorentzian manifold with differentiable boundary $\partial \mathscr{S}=\partial \mathscr{D} \times \mathbb{R}$ if a stationary Lorentzian manifold $(\mathscr{L}, g)$, with $\mathscr{L}=\mu \times \mathbb{R}$, exists such that $\mathscr{D}$ is an open domain of $\mathcal{M}, g$ restricted to $\mathscr{S}$ is $\langle\cdot, \cdot\rangle_{L}$, and $\overline{\mathscr{D}}$ is a complete manifold with differentiable boundary.

Remark that if $\mathscr{Y}$ is a stationary Lorentzian manifold with convex boundary, since $\partial \mathscr{D}$ is differentiable, there exists a smooth function $\phi: \overline{\mathscr{D}} \rightarrow \mathbb{R}$ satisfying

$$
\begin{gathered}
\phi^{-1}(0)=\partial \mathscr{D}, \\
\phi>0 \quad \text { on } \mathscr{D}, \\
\nabla \phi(x) \neq 0 \quad \forall x \in \partial \mathscr{D} .
\end{gathered}
$$

Moreover, $\Phi$ can be chosen such that, for any $z=(x, t) \in \mathscr{L}$,

$$
\Phi(z)=\Phi(x, t)=\phi(x)
$$


and then

$$
\nabla^{L} \Phi(z)=(\nabla \phi(x), 0)
$$

We prove the following theorem.

THEOREM 1.6. Let $\left(\mathscr{S},\langle\cdot, \cdot\rangle_{L}\right)$ be a stationary Lorentzian manifold with differentiable and convex boundary; assume that the assumptions of Theorem 1.1 hold and that

$$
\left\langle\mathscr{F}(y)[\zeta], \nabla^{L} \Phi(y)\right\rangle \leq 0 \quad \forall y \in \partial \mathscr{S}, \zeta \in T_{y} \partial \mathscr{S} .
$$

Then, for each two given events in 9 a trajectory $\gamma$ joining them exists. Moreover, if $\mathscr{D}$ is noncontractible in itself, then, for each two given events of $\mathscr{S}$ a sequence $\left\{\gamma_{m}\right\}$ of trajectories joining them exists.

EXAMPLE 1.7. We consider an open subset of the Minkowski spacetime. This spacetime is the model of special relativity which describes situations in which the gravitational effects are negligible. Given the vector field $A$, the 2-form $\operatorname{curl} A$ can be written as

$$
\operatorname{curl} A=\sum_{i=1}^{3} E^{i} d x^{i} \wedge d t+B^{1} d x^{2} \wedge d x^{3}+B^{2} d x^{3} \wedge d x^{1}+B^{3} d x^{1} \wedge d x^{2}
$$

where $E^{i}, B^{i}, i=1,2,3$, are differentiable functions. This 2-form, or the associated endomorphism field $\mathscr{F}$, is called the electromagnetic field. Moreover, $E=\sum_{i=1}^{3} E^{i} \partial_{i}$ is the electric field and $B=\sum_{i=1}^{3} B^{i} \partial_{i}$ the magnetic field. These concepts can be extended to the tangent space of any Lorentzian manifold, whenever a timelike tangent vector (which plays the role of $\partial_{t}$ ) is fixed (see, e.g., [17, page 75]). Thus, hypothesis (1.15) only involves the magnetic field naturally associated to the decomposition $\mathscr{S}=\mathscr{D} \times \mathbb{R}$.

The paper is organized as follows. In Section 2, we state a variational principle which allows us to overcome the problems arising in the study of $F$ because of the indefiniteness of the metric. Then in Section 3 we prove Theorem 1.1 by using classical critical point theory. Finally, in Section 4, thanks to a penalization technique (necessary in order to find trajectories not touching the boundary), we demonstrate Theorem 1.6.

2. A variational principle for trajectories. From now on, we assume that an $M$ is a submanifold of $\left(\mathbb{R}^{N},\langle\cdot, \cdot\rangle\right)$, for $N$ sufficiently large. Thus (see [16])

$$
H^{1}([0,1], \mathcal{M})=\left\{y \in H^{1}\left([0,1], \mathbb{R}^{N}\right) \mid y([0,1]) \subset \mathcal{M}\right\}
$$


is a submanifold of the Sobolev space $H^{1}\left([0,1], \mathbb{R}^{N}\right)$. Fix $z=\left(p, t_{1}\right), w=$ $\left(q, t_{2}\right) \in \mathscr{L}$ and consider the product manifold

$$
\mathscr{L}=\Omega^{1}(\mathcal{M}) \times H^{1}\left(t_{1}, t_{2}\right)
$$

where

$$
\begin{aligned}
& \Omega^{1}(\mathcal{M})=\left\{y \in H^{1}([0,1], \mathcal{M}) \mid y(0)=p, y(1)=q\right\}, \\
& H^{1}\left(t_{1}, t_{2}\right)=\left\{t \in H^{1}([0,1], \mathbb{R}) \mid t(0)=t_{1}, t(1)=t_{2}\right\} .
\end{aligned}
$$

We recall that for any $y \in H^{1}([0,1], \mathcal{M})$, the tangent space at $H^{1}([0,1], \mathcal{M})$ is given by

$$
T_{y} H^{1}([0,1], \mathcal{M})=\left\{v \in H^{1}\left([0,1], \mathbb{R}^{N}\right) \mid v(s) \in T_{y(s)} \mathcal{M} \forall s \in[0,1]\right\}
$$

(see [16]) and for any $t \in H^{1}\left(t_{1}, t_{2}\right)$ the tangent space at $H^{1}\left(t_{1}, t_{2}\right)$ is

$$
H_{0}^{1}\left([0,1], \mathbb{R}^{N}\right)=\left\{y \in H^{1}\left([0,1], \mathbb{R}^{N}\right) \mid y(0)=0=y(1)\right\}
$$

We will consider on $\mathscr{E}$ the functional $F$ in (1.6) given explicitly by

$$
\begin{aligned}
F(\gamma)= & \int_{0}^{1}\left[\langle\dot{x}, \dot{x}\rangle+2\langle\delta(x), \dot{x}\rangle \dot{t}-\beta(x) \dot{t}^{2}\right] d s \\
& +\int_{0}^{1}\left[\left\langle A_{1}(x), \dot{x}\right\rangle+\langle\delta(x), \dot{x}\rangle A_{2}(x)\right] d s \\
& +\int_{0}^{1}\left[\left\langle\delta(x), A_{1}(x)\right\rangle \dot{t}-\beta(x) A_{2}(x) \dot{t}\right] d s .
\end{aligned}
$$

Integration by parts and a boot-strap argument show that the critical points of $F$ are smooth, (see [9, Proposition 6.1]). The following lemma holds (see [5, Section 2] and [2, Lemma 2.1]).

LEMMA 2.1. Let $\gamma \in \mathscr{L}$. Then $\gamma$ satisfies (1.4) if and only if it is a critical point of F on $\mathscr{L}$.

REMARK 2.2. The first equation in (1.4) or equivently

$$
-2 D_{s} \dot{\gamma}+\left(\nabla^{L} A(\gamma)\right)^{T} \dot{\gamma}-\nabla^{L} A(\gamma) \dot{\gamma}=0,
$$


where $\nabla^{L} A$ denotes the gradient of the vector field $A$ and $\left(\nabla^{L} A\right)^{T}$ its transpose, has a prime integral, in fact

$$
\frac{d}{d s}\langle\dot{\gamma}, \dot{\gamma}\rangle_{L}=2\left\langle D_{s} \dot{\gamma}, \dot{\gamma}\right\rangle_{L}=\left\langle\left(\nabla^{L} A(\gamma)\right)^{T} \dot{\gamma}-\nabla^{L} A(\gamma) \dot{\gamma}, \dot{\gamma}\right\rangle_{L}=0 .
$$

We recall that in [5] a new variational principle for the fundamental equations of the classical physics has been introduced; such a principle allows one to obtain a sort of unification of the gravitational and the electromagnetic fields. The basic point of this variational principle is that the world-line of a material point is parametrized by a parameter $s$ which carries some physical information, namely it is related to the rest mass and to the charge. In particular, the inertial mass turns out to be a constant of the motion, which is determined by the initial conditions and also the equality between the inertial and gravitational mass can be deduced.

By Lemma 2.1, to find trajectories joining two events, we have to investigate the existence of the critical points of functional (1.6) on $\mathscr{E}$. Classical minimization arguments cannot be applied to functional $F$ since it is strongly indefinite (i.e., it is unbounded both from above and from below and the Morse index of its critical points is $+\infty$ ). As for the geodesic problem (see, e.g., [11]), when we deal with stationary manifolds and stationary vector fields, a variational principle can be proved. This variational principle (see [1] for the details) reduces the study of the critical points of $F$ to the search of the critical points of a functional which is bounded from below under our assumptions on the coefficients of the metric and on the vector field. Remark that for any $x \in \Omega^{1}(\mathcal{M})$ the functional $F(x, \cdot)$ has on $H^{1}\left(t_{1}, t_{2}\right)$ one and only one critical point, say that $t=\Psi(x)$ (where $\Psi$ can be explicitly determinated). Consider on $\Omega^{1}(\mathcal{M})$ the functional

$$
J(x)=F(x, \Psi(x))
$$

which is smooth by the implicit function theorem and whose first variation is given by

$$
J^{\prime}(x)[\xi]=F_{x}(x, \Psi(x))[\xi],
$$

(where $F_{x}$ denotes the partial derivative of $F$ with respect to $x$ ). Thus we get the following result.

THEOREM 2.3. Let $\gamma=(x, t) \in \mathscr{L}$. The following propositions are equivalent:

(a) $\gamma$ is a critical point of $F$;

(b) (i) $x$ is the critical point of J;

(ii) $t=\Psi(x)$.

Moreover, if (a) or (b) is true,

$$
F(\gamma)=J(x) .
$$


The functional $J$ can be explicitly evaluated and it results that

$$
\begin{aligned}
J(x)= & \int_{0}^{1}\langle\dot{x}, \dot{x}\rangle d s+\int_{0}^{1} \frac{\langle\delta(x), \dot{x}\rangle^{2}}{\beta(x)} d s+\frac{1}{4} \int_{0}^{1} \frac{\left\langle\delta(x), A_{1}(x)\right\rangle^{2}}{\beta(x)} d s \\
& +\int_{0}^{1}\left\langle A_{1}(x), \dot{x}\right\rangle d s+\int_{0}^{1} \frac{\left\langle\delta(x), A_{1}(x)\right\rangle\langle\delta(x), \dot{x}\rangle}{\beta(x)} d s \\
& -\frac{1}{2} \int_{0}^{1}\left\langle\delta(x), A_{1}(x)\right\rangle A_{2}(x) d s \\
& +\frac{1}{4} \int_{0}^{1} A_{2}^{2}(x) \beta(x) d s-\frac{K^{2}(x)}{4} \int_{0}^{1} \frac{1}{\beta(x)} d s,
\end{aligned}
$$

where

$K(x)=\frac{2 \Delta-2 \int_{0}^{1}(\langle\delta(x), \dot{x}\rangle / \beta(x)) d s-\int_{0}^{1}\left(\left\langle\delta(x), A_{1}(x)\right\rangle / \beta(x)\right) d s+\int_{0}^{1} A_{2}(x) d s}{\int_{0}^{1}(1 / \beta(x)) d s}$,

with $\Delta=t_{2}-t_{1}$.

3. Proof of Theorem 1.1. By Lemma 2.1 and Theorem 2.3, we have to study the critical points of the functional (2.12) on $\Omega^{1}(\mathcal{M})$.

REMARK 3.1. The assumptions of Theorem 1.1 imply, by using the Hölder inequality, that

$$
J(x) \geq \int_{0}^{1}\langle\dot{x}, \dot{x}\rangle d s-C_{1} \int_{0}^{1} \sqrt{\langle\dot{x}, \dot{x}\rangle} d s-C_{2},
$$

where

$$
\begin{aligned}
& C_{1}=a_{1}+\frac{d^{2} a_{1}}{\eta}+\frac{d^{2} a_{1}}{\eta^{2}}+\frac{d a_{2}}{\eta}+\frac{2 \Delta d}{\eta}, \\
& C_{2}=\frac{d a_{1} a_{2}}{2}+\Delta^{2}+a_{2} \Delta+\frac{d a_{1} a_{2}}{2 \eta}+\frac{a_{2}^{2}}{2}+\frac{\Delta d a_{1}}{\eta},
\end{aligned}
$$

hence the functional $J$ is bounded from below (we assume $b=1$ ).

Before proving Theorem 1.1, we recall some definitions. If $(X, h)$ is a Riemannian manifold modelled on a Hilbert space and $f \in \mathscr{C}^{1}(X, \mathbb{R}), f$ satisfies the Palais-Smale condition if every sequence $\left\{y_{m}\right\}$ such that

$$
\begin{gathered}
\left\{f\left(y_{m}\right)\right\} \text { is bounded, } \\
\left\|\nabla f\left(y_{m}\right)\right\| \longrightarrow 0,
\end{gathered}
$$

contains a converging subsequence, where $\nabla f(y)$ denotes the gradient of $f$ at the point $y$ with respect to the metric $h$ and $\|\cdot\|$ is the norm on the tangent bundle induced by $h$. By standard arguments, it can be proved that $J$ satisfies the Palais-Smale condition, (see, e.g., [1, Proposition 3.5]). The category, 
denoted with cat ${ }_{X} Y$, of a subspace $Y$ of a topological space $X$ is the least number of closed and contractible subset of $X$ covering $Y$. If $Y$ is not covered by a finite number of such subsets of $X$, we set cat ${ }_{X} Y=+\infty$.

Proof of TheOrem 1.1. As $J$ is bounded from below and satisfies the Palais-Smale condition on $\Omega^{1}(\mathcal{M})$, it admits a minimum point $x$ which corresponds to a critical point $\gamma=(x, \Psi(x))$ of $F$ by virtue of Theorem 2.3. By Lemma 2.1, the proof of (i) is complete. Moreover, by a result of Fadell and Husseini (see [10]), the Ljusternik-Schnirelman category of $\Omega^{1}(\mathcal{M})$ is infinite. Since

$$
\operatorname{cat}_{\Omega^{1}(\mathcal{M})} J^{c}<+\infty \quad \forall c \in \mathbb{R},
$$

(where $J^{c}=\left\{x \in \Omega^{1}(\mathcal{M}) \mid J(x) \leq c\right\}$ ), by classical arguments of LjusternikSchnirelman critical point theory, we get the existence of a sequence $\left\{x_{m}\right\}$ of critical points of $J$ such that

$$
\lim _{m \rightarrow+\infty} J\left(x_{m}\right)=+\infty .
$$

Hence, set $\gamma_{m}=\left(x_{m}, \Psi\left(x_{m}\right)\right)$ for any $m \in \mathbb{N}$, by Theorem 2.3 and Lemma 2.1, we get the existence of infinitely many trajectories joining the two given events such that

$$
\lim _{m \rightarrow+\infty} F\left(\gamma_{m}\right)=+\infty
$$

4. Proof of Theorem 1.6. When we deal with open subsets $\mathscr{Y}$ of $\mathscr{L}$, we need to penalize functionals $F$ and $J$ because Palais-Smale sequences converging to a critical point touching the boundary $\partial \mathscr{S}$ could exist. We consider for any $\epsilon \in] 0,1]$ the functionals

$$
\begin{aligned}
& F_{\epsilon}(\gamma)=F(\gamma)+\int_{0}^{1} \psi_{\epsilon}\left(\frac{1}{\Phi^{2}(\gamma)}\right) d s, \\
& J_{\epsilon}(x)=J(x)+\int_{0}^{1} \psi_{\epsilon}\left(\frac{1}{\phi^{2}(x)}\right) d s,
\end{aligned}
$$

respectively, on $\Omega^{1}(\mathscr{D}) \times H^{1}\left(t_{1}, t_{2}\right)$ and $\Omega^{1}(\mathscr{D})$, where $\Phi$ is as in (1.9), $\phi$ is as in (1.12), and $\left(\psi_{\epsilon}\right)_{\epsilon \in] 0,1]}$ is a family of nonnegative increasing functions in $\mathscr{C}^{2}(\mathbb{R}, \mathbb{R})$ such that

$$
\begin{gathered}
\psi_{\epsilon}(s)=0 \quad \text { if } s \leq \frac{1}{\epsilon}, \\
\lim _{s \rightarrow \infty} \psi_{\epsilon}(s)=\infty, \\
\psi_{\epsilon}(s) \geq a_{\epsilon} s-b_{\epsilon}
\end{gathered}
$$

for some $a_{\epsilon}>0, b_{\epsilon} \geq 0$. We point out that the variational principle stated in Theorem 2.3 still holds since the penalizating term does not depend on $t$. The 
following lemma (see, e.g., [13]) plays a basic role in our penalization technique. We denote by $\|\cdot\|_{2}$ the usual norm in $L^{2}\left([0,1], \mathbb{R}^{N}\right)$.

LEMMA 4.1. Let $\left\{x_{m}\right\}$ be a sequence in $\Omega^{1}(\mathscr{D})$ such that

$$
\sup _{m \in \mathbb{N}}\left\|\dot{x}_{m}\right\|_{2}<+\infty
$$

and let $\left\{s_{m}\right\}$ be a sequence in $[0,1]$ such that

$$
\lim _{m \rightarrow+\infty} \phi\left(x_{m}\left(s_{m}\right)\right)=0 .
$$

Then

$$
\lim _{m \rightarrow+\infty} \int_{0}^{1} \psi_{\epsilon}\left(\frac{1}{\phi^{2}\left(x_{m}\right)}\right) d s=+\infty
$$

LEMMA 4.2. For any $\epsilon \in] 0,1]$, let $\left\{x_{m}\right\}$ be a sequence in $\Omega^{1}(\mathscr{D})$ such that

$$
J_{\epsilon}\left(x_{m}\right) \leq C \quad \forall m \in \mathbb{R}
$$

for $a C \in \mathbb{R}$. Then

$$
d=\inf \left\{\phi\left(x_{m}(s)\right) \mid m \in \mathbb{N}, s \in I\right\}>0 .
$$

Proof. By (4.6) and the form of the penalization, we get

$$
J\left(x_{m}\right) \leq C \quad \forall m \in \mathbb{R} .
$$

Then, by (2.12) and the assumptions of Theorem 1.1, it results that

$$
\left\|\dot{x}_{m}\right\|_{2}^{2} \leq C+\left(a_{1}+\frac{d^{2}}{\eta} a_{1}+\frac{2}{\eta} d\right)\left\|\dot{x}_{m}\right\|_{2}+c
$$

for a suitable $c \in \mathbb{R}$. Thus $\left\{\left\|\dot{x}_{m}\right\|_{2}\right\}$ is bounded and the proof follows by Lemma 4.1.

We omit the proof of the following proposition since it is a combination of the proof of [11, Theorem 3.3] and [4, Lemma 4.3].

Proposition 4.3. Let $J_{\epsilon}$ be as in (4.1). Then

(i) for any $\epsilon \in] 0,1]$ and for any $c \in \mathbb{R}$, the sublevels

$$
J_{\epsilon}^{c}=\left\{x \in \Omega^{1}(\mathscr{D}) \mid J_{\epsilon}(x) \leq c\right\}
$$

are complete metric subspaces of $\Omega^{1}(\mathscr{D})$;

(ii) for any $\epsilon \in] 0,1], J_{\epsilon}$ satisfies the Palais-Smale condition. 
By the previous proposition and Remark 3.1, there exists a family $\left\{x_{\epsilon}\right\}$ of critical points of $J_{\epsilon}$ satisfying (4.6). Thus, by Theorem 2.3, set $\gamma_{\epsilon}=\left(x_{\epsilon}, \Psi\left(x_{\epsilon}\right)\right)$, we find a family $\left\{\gamma_{\epsilon}\right\}$ of critical points of $F_{\epsilon}$ such that

$$
\left.\left.F_{\epsilon}\left(\gamma_{\epsilon}\right) \leq C \quad \forall \epsilon \in\right] 0,1\right] .
$$

REMARK 4.4. It is easy to prove that a critical point $\gamma$ of $F_{\epsilon}$ satisfies the following equation:

$$
D_{s} \dot{\gamma}=\frac{1}{2} \mathscr{F}_{\mathcal{F}}(\gamma)[\dot{\gamma}]-\frac{2}{\Phi^{3}(\gamma)} \psi_{\epsilon}^{\prime}\left(\frac{1}{\Phi^{2}(\gamma)}\right) \nabla^{L} \Phi(\gamma)
$$

Thus, multiplying by $\dot{\gamma}$, we get the existence of $H_{\epsilon}(\gamma) \in \mathbb{R}$ such that

$$
H_{\epsilon}(\gamma)=\langle\dot{\gamma}, \dot{\gamma}\rangle_{L}-\psi_{\epsilon}\left(\frac{1}{\Phi^{2}(\gamma)}\right)
$$

We set for any $\epsilon \in] 0,1], s \in[0,1]$,

$$
\mu_{\epsilon}(s)=\frac{2}{\Phi^{3}(\gamma(s))} \psi_{\epsilon}^{\prime}\left(\frac{1}{\Phi^{2}(\gamma(s))}\right)
$$

The following estimate on the family $\left\{\mu_{\epsilon}\right\}$ holds.

LEMmA 4.5. There exists $\left.\left.\epsilon_{0} \in\right] 0,1\right]$ such that the family of functions $\left(\mu_{\epsilon}\right)_{\left.\epsilon \in] 0, \epsilon_{0}\right]}$ is bounded in $\mathscr{C}([0,1], \mathbb{R})$.

Proof. For any $\epsilon \in] 0,1]$ and $s \in[0,1]$, we set $u_{\epsilon}(s)=\Phi\left(\gamma_{\epsilon}(s)\right)$, so $u_{\epsilon}$ is a $\mathscr{C}^{2}$ function on $[0,1]$. Let $s_{\epsilon}$ be a minimum point for $u_{\epsilon}$. Since $\psi_{\epsilon}$ is convex, $\psi_{\epsilon}^{\prime}$ is nondecreasing, thus it results that

$$
\mu_{\epsilon}(s) \leq \mu_{\epsilon}\left(s_{\epsilon}\right) \quad \forall s \in[0,1] .
$$

Hence, it is enough to prove that $\left\{\mu_{\epsilon}\left(s_{\epsilon}\right)\right\}$ is bounded and to study the case in which

$$
\inf _{\epsilon \in] 0,1]} \Phi\left(\gamma_{\epsilon}\left(s_{\epsilon}\right)\right)=0 .
$$

Differentiating twice, we get

$$
\begin{aligned}
0 \leq \ddot{u}_{\epsilon}\left(s_{\epsilon}\right)= & H_{\Phi}^{L}\left(\gamma_{\epsilon}\left(s_{\epsilon}\right)\right)\left[\dot{\gamma}_{\epsilon}\left(s_{\epsilon}\right), \dot{\gamma}_{\epsilon}\left(s_{\epsilon}\right)\right] \\
& \left.+\left\langle\nabla^{L} \Phi\left(\gamma_{\epsilon}\left(s_{\epsilon}\right)\right), \frac{1}{2} \mathscr{F}_{(\gamma}\left(s_{\epsilon}\right)\right)\left[\dot{\gamma}\left(s_{\epsilon}\right)\right]\right\rangle_{L} \\
& -\mu_{\epsilon}\left(s_{\epsilon}\right)\left|\nabla^{L} \Phi\left(\gamma_{\epsilon}\left(s_{\epsilon}\right)\right)\right|^{2} .
\end{aligned}
$$


We consider on $\mathscr{L}$ the Riemannian metric given by

$$
\langle\zeta, \zeta\rangle_{R}=\langle\xi, \xi\rangle+\beta(x) \tau^{2}
$$

for any $\gamma=(x, t) \in \mathscr{L}$ and $\zeta=(\xi, \tau) \in T_{\gamma} \mathscr{L}$. As $H_{L}^{\Phi}$ is a bilinear form,

$$
H_{L}^{\Phi}\left(\gamma_{\epsilon}\left(s_{\epsilon}\right)\right)\left[\dot{\gamma}_{\epsilon}\left(s_{\epsilon}\right), \dot{\gamma}_{\epsilon}\left(s_{\epsilon}\right)\right] \leq c_{1}\left\langle\dot{\gamma}_{\epsilon}\left(s_{\epsilon}\right), \dot{\gamma}_{\epsilon}\left(s_{\epsilon}\right)\right\rangle_{R}
$$

for some $c_{1}>0$. Moreover, as 0 is a regular value for $\Phi$, for $\epsilon$ sufficiently small,

$$
\left|\nabla^{L} \Phi\left(\gamma_{\epsilon}\left(s_{\epsilon}\right)\right)\right|^{2} \geq c_{2}
$$

for some $c_{2}>0$. Thus, by (4.17) and (1.15) follows, for $\epsilon$ sufficiently small,

$$
c_{2} \mu_{\epsilon}\left(s_{\epsilon}\right) \leq c_{1}\left(\left\langle x_{\epsilon}\left(s_{\epsilon}\right), x_{\epsilon}\left(s_{\epsilon}\right)\right\rangle+\beta\left(x_{\epsilon}\left(s_{\epsilon}\right)\right) \dot{t}_{\epsilon}^{2}\left(s_{\epsilon}\right)\right) .
$$

By (4.13), it results that

$$
\begin{aligned}
c_{2} \mu_{\epsilon}\left(s_{\epsilon}\right) \leq & c_{1}\left|H_{\epsilon}\left(\gamma_{\epsilon}\right)\right|+2 c_{1} \beta\left(x_{\epsilon}\left(s_{\epsilon}\right)\right) \dot{t}_{\epsilon}^{2}\left(s_{\epsilon}\right) \\
& +2 c_{1}\left|\left\langle\delta\left(x_{\epsilon}\left(s_{\epsilon}\right)\right), \dot{x}_{\epsilon}\left(s_{\epsilon}\right)\right\rangle\right|+c_{1} \psi_{\epsilon}\left(\frac{1}{\Phi^{2}\left(\gamma_{\epsilon}\left(s_{\epsilon}\right)\right)}\right) .
\end{aligned}
$$

By Remark 3.1 and (4.6), it is easy to verify that

$$
\left|H_{\epsilon}\left(\gamma_{\epsilon}\right)\right| \leq c_{3}
$$

for some $c_{3}>0$, so that

$$
c_{2} \mu_{\epsilon}\left(s_{\epsilon}\right) \leq c_{4}+c_{1} \psi_{\epsilon}\left(\frac{1}{\Phi^{2}\left(\gamma_{\epsilon}\left(s_{\epsilon}\right)\right)}\right)
$$

for some $c_{4}>0$. Since

$$
\left.\left.\psi_{\epsilon}(s) \leq \psi_{\epsilon}^{\prime}(s) \quad \forall \epsilon \in\right] 0,1\right], s \in[0,1]
$$

we get

$$
\mu_{\epsilon}\left(s_{\epsilon}\right) \leq c_{5}+c_{6} \psi_{\epsilon}^{\prime}\left(\frac{1}{\Phi^{2}\left(\gamma_{\epsilon}\left(s_{\epsilon}\right)\right)}\right)
$$

for some $c_{5}, c_{6}>0$. From (4.16), for a $\left.\sigma \in\right] 0,1[$ and $\epsilon$ small enough,

$$
\Phi\left(\gamma_{\epsilon}\left(s_{\epsilon}\right)\right)<\frac{\sigma}{c_{6}}
$$


hence we get

$$
\mu_{\epsilon}\left(s_{\epsilon}\right) \leq c_{5}+\sigma \mu_{\epsilon}\left(s_{\epsilon}\right)
$$

and the proof is complete.

The same arguments used in [11, Lemma 4.7] allow us to obtain the following proposition.

Proposition 4.6. Let $\left\{\gamma_{\epsilon}\right\}$ be a family in $\mathscr{E}$ such that for any $\left.\left.\epsilon \in\right] 0,1\right], \gamma_{\epsilon}$ is a critical point of $F_{\epsilon}$ and (4.6) holds. Then there exist an infinitesimal and decreasing sequence $\left\{\epsilon_{m}\right\}$ in $\left.] 0,1\right]$ and a curve $\gamma=(x, t) \in H^{1}([0,1], \bar{D}) \times$ $H^{1}\left(t_{1}, t_{2}\right)$ such that

$$
\gamma_{\epsilon_{m}} \rightarrow \gamma \quad \text { in } H^{1}\left([0,1], \mathbb{R}^{N}\right) \times H^{1}([0,1], \mathbb{R})
$$

Proof of TheOrem 1.6. Standard arguments show that the curve $\gamma$ found in Proposition 4.6 belongs to $H^{2}\left([0,1], \mathbb{R}^{N}\right) \times H^{2}([0,1], \mathbb{R})$ and that it solves the equation

$$
D_{s} \dot{\gamma}(s)=\mu(s) \nabla^{L} \Phi(\gamma(s))+\frac{1}{2} \mathscr{F}(\gamma(s))[\dot{\gamma}(s)],
$$

where $\mu \in L^{2}([0,1], \mathbb{R})$ is positive almost everywhere in $[0,1]$ and vanishes if $\gamma(s) \in \mathscr{Y}$. If $\left.s_{0} \in\right] 0,1\left[\right.$ is such that $\gamma\left(s_{0}\right) \in \partial \mathscr{Y}(\gamma(0)=z, \gamma(1)=w \in \mathscr{D})$, and $D_{s} \dot{\gamma}\left(s_{0}\right)$ exists, set $u(s)=\Phi(\gamma(s))$ we get

$$
\begin{aligned}
0 \leq \ddot{u}\left(s_{0}\right)= & H_{\Phi}^{L}\left(\gamma\left(s_{0}\right)\right)\left[\dot{\gamma}\left(s_{0}\right), \dot{\gamma}\left(s_{0}\right)\right] \\
& +\left\langle\nabla^{L} \Phi\left(\gamma\left(s_{0}\right)\right), \frac{1}{2} \mathscr{F}\left(\gamma\left(s_{0}\right)\right)\left[\dot{\gamma}\left(s_{0}\right)\right]\right\rangle_{L} \\
& -\mu\left(s_{0}\right)\left|\nabla^{L} \Phi\left(\gamma\left(s_{0}\right)\right)\right|^{2} .
\end{aligned}
$$

Thus by (1.15) and the convexity of $\partial \mathscr{S}$

$$
\mu\left(s_{0}\right)\left|\nabla^{L} \Phi\left(\gamma\left(s_{0}\right)\right)\right|^{2} \leq 0
$$

and this implies that $\mu\left(s_{0}\right)=0$. Moreover, it can be proved that if $s_{0} \in[0,1]$ is such that $\gamma\left(s_{0}\right) \in \mathscr{Y}$, there exists a neighborhood $I$ of $s_{0}$ such that $u(s)=0$ for every $s \in I$. Thus $\gamma$ is a trajectory joining $z$ and $w$. Now, it suffices to prove that the range of $\gamma$ is contained in $\mathscr{S}$. Let $C=\{s \in[0,1] \mid \gamma(s) \in \partial \mathscr{Y}\}$ and assume that $C$ is nonempty. Clearly, $C$ is compact; say $s_{M} \in$ ]0,1[ its maximum. Using 
the Gronwall lemma, we prove that there exists $\sigma>0$ such that $\left[s_{M}, s_{M}+\sigma\right] \subset$ $C$, getting a contradiction. Indeed, for a $\eta_{1}>0$, as $\gamma\left(s_{M}\right) \in \partial \mathscr{S}$, there exists $\sigma>0$ such that

$$
\Phi(\gamma(s))<\eta_{1} \quad \forall s \in\left[s_{M}, s_{M}+\sigma\right]
$$

and we can consider the projection $\gamma_{p}=\left(x_{p}, t_{p}\right):\left[s_{M}, s_{M}+\sigma\right] \rightarrow \partial \mathscr{Y}$ of $\gamma$ on $\partial \mathscr{Y}$ obtained by using the flow of the vector field $-\nabla \Phi /|\nabla \Phi|^{2}$. From (1.10), we get

$$
H_{\Phi}^{L}\left(\gamma_{p}(s)\right)\left[\dot{\gamma}_{p}(s), \dot{\gamma}_{p}(s)\right] \leq 0 \quad \forall s \in\left[s_{M}, s_{M}+\sigma\right]
$$

Consider $u$ as before; then for any $s \in\left[s_{M}, s_{M}+\sigma\right]$

$$
\begin{aligned}
\ddot{u}(s) \leq & H_{\Phi}^{L}(\gamma(s))[\dot{\gamma}(s), \dot{\gamma}(s)]-H_{\Phi}^{L}\left(\gamma_{p}(s)\right)\left[\dot{\gamma}_{p}(s), \dot{\gamma}_{p}(s)\right] \\
& +\frac{1}{2}\left\langle\nabla^{L} \Phi(\gamma(s)), \mathscr{F}(\gamma(s))[\dot{\gamma}(s)]\right\rangle_{L} .
\end{aligned}
$$

Reasoning as in [3, Theorem 4.3], it results that

$$
H_{\Phi}^{L}(\gamma(s))[\dot{\gamma}(s), \dot{\gamma}(s)]-H_{\Phi}^{L}\left(\gamma_{p}(s)\right)\left[\dot{\gamma}_{p}(s), \dot{\gamma}_{p}(s)\right] \leq M_{1} u(s)+M_{2} \dot{u}(s)
$$

for any $s \in\left[s_{M}, s_{M}+\sigma\right]$ for some $M_{1}, M_{2} \in \mathbb{R}$. Moreover, by (1.15)

$$
\begin{aligned}
&\left\langle\nabla^{L} \Phi(\gamma(s)), \mathscr{F}(\gamma(s))[\dot{\gamma}(s)]\right\rangle_{L} \\
& \leq\left\langle\nabla^{L} \Phi(\gamma(s)), \mathscr{F}(\gamma(s))[\dot{\gamma}(s)]\right\rangle_{L} \\
&-\left\langle\nabla^{L} \Phi\left(\gamma_{p}(s)\right), \mathscr{F}\left(\gamma_{p}(s)\right)\left[\dot{\gamma}_{p}(s)\right]\right\rangle_{L} \\
&=\left\langle\nabla^{L} \Phi(\gamma(s)), \mathscr{F}(\gamma(s))[\dot{\gamma}(s)]\right\rangle_{L} \\
&-\left\langle\nabla^{L} \Phi\left(\gamma_{p}(s)\right), \mathscr{F}(\gamma(s))[\dot{\gamma}(s)]\right\rangle_{L} \\
&+\left\langle\nabla^{L} \Phi\left(\gamma_{p}(s)\right), \mathscr{F}(\gamma(s))[\dot{\gamma}(s)]\right\rangle_{L} \\
&-\left\langle\nabla^{L} \Phi\left(\gamma_{p}(s)\right), \mathscr{F}\left(\gamma_{p}(s)\right)\left[\dot{\gamma}_{p}(s)\right]\right\rangle_{L} .
\end{aligned}
$$

As $\mathscr{F}$ and $\phi$ are $\mathscr{C}^{2}, M_{3}>0$ exists such that

$$
\left\langle\nabla^{L} \Phi(\gamma(s)), \mathscr{F}(\gamma(s))[\dot{\gamma}(s)]\right\rangle_{L} \leq M_{3}\left|x(s)-x_{p}(s)\right| \leq M_{3} u(s)
$$

for an $M_{3} \in \mathbb{R}$. Thus

$$
\ddot{u}(s) \leq\left(M_{1}+M_{3}\right) u(s)+M_{2} \dot{u}(s) \quad \forall s \in\left[s_{M}, s_{M}+\sigma\right] .
$$


Since $u\left(s_{M}\right)=0$ and $\dot{u}\left(s_{M}\right)=0$ by the Gronwall lemma, we obtain $u \equiv 0$ in $\left[s_{M}, s_{M}+\sigma\right]$. Now, assume that $\mathscr{D}$ is not contractible in itself. Set for any $c \in \mathbb{R}$

$$
J_{c}=\left\{x \in \Omega^{1}(\mathscr{D}) \mid J(x) \geq c\right\}, \quad J_{\epsilon, c}=\left\{x \in \Omega^{1}(\mathscr{D}) \mid J_{\epsilon}(x) \geq c\right\} .
$$

It can be proved that even if $J$ does not satisfy the Palais-Smale condition,

$$
\operatorname{cat}_{\Omega^{1}(\mathscr{D})} J^{c}<+\infty
$$

(see [8, Lemma 4.3]). Then by the Fadell and Husseini result and classical arguments there exists $m \in \mathbb{N}$ such that

$$
X \cap J_{c} \neq \varnothing
$$

for any $X \in \Gamma_{m}=\left\{Y \subset \Omega^{1}(\mathscr{D}) \mid \operatorname{cat}_{\Omega^{1}(\mathfrak{D})} Y \geq m\right\}$. Since $J_{c} \subset J_{\epsilon, c}$, for any $X \in \Gamma_{m}$ it also results that

$$
\left.\left.X \cap J_{\epsilon, c} \neq \varnothing \quad \forall \epsilon \in\right] 0,1\right] .
$$

By Proposition 4.3 , for any $m \in \mathbb{N}, \epsilon \in] 0,1]$, the values

$$
c_{\epsilon, m}=\inf _{X \in \Gamma_{m}} \sup _{x \in X} J_{\epsilon}(x)
$$

are well defined and are critical values of $J_{\epsilon}$. Thus we obtain

$$
\left.\left.c \leq c_{\epsilon, m} \quad \forall \epsilon \in\right] 0,1\right] .
$$

Since the singular homology has compact support, then there exists a compact $C \in \Gamma_{m}$. Therefore,

$$
\left.\left.c \leq c_{\epsilon, m} \leq \max J_{\epsilon}(C) \leq \max J_{1}(C) \quad \forall \epsilon \in\right] 0,1\right] .
$$

Reasoning as in the first part of the proof, we get the existence of a critical point of $J$. Moreover,

$$
J(x) \geq c
$$

(see [8, Theorem 1.9]), thus being $c$ arbitrary the thesis follows. 


\section{REFERENCES}

[1] R. Bartolo, Trajectories on stationary Lorentzian manifolds, preprint, Universidad de Granada, 1997.

[2] _ , Trajectories connecting two events of a Lorentzian manifold in the presence of a vector field, J. Differential Equations 153 (1999), no. 1, 82-95.

[3] R. Bartolo, A. Germinario, and M. Sánchez, A note on the boundary of a static Lorentzian manifold, Differential Geom. Appl. 16 (2002), no. 2, 121-131.

[4] R. Bartolo, E. Mirenghi, and M. Tucci, Periodic trajectories on Lorentz manifolds under the action of a vector field, J. Differential Equations 166 (2000), no. 2, 478-500.

[5] V. Benci and D. Fortunato, A new variational principle for the fundamental equations of classical physics, Found. Phys. 28 (1998), no. 2, 333-352.

[6] V. Benci, D. Fortunato, and F. Giannoni, On the existence of multiple geodesics in static space-times, Ann. Inst. H. Poincaré Anal. Non Linéaire 8 (1991), no. 1, 79-102.

[7] _ Geodesics on static Lorentz manifolds with convex boundary, Progress in Variational Methods in Hamiltonian Systems and Elliptic Equations (L'Aquila, 1990), Pitman Res. Notes Math. Ser., vol. 243, Longman Sci. Tech., Harlow, 1992, pp. 21-41.

[8] _ On the existence of geodesics in static Lorentz manifolds with singular boundary, Ann. Scuola Norm. Sup. Pisa Cl. Sci. (4) 19 (1992), no. 2, 255289.

[9] E. Caponio and A. Masiello, Trajectories for relativistic particles under the action of an electromagnetic field in a stationary space-time, Nonlinear Anal., Ser. A: Theory Methods 50 (2002), no. 1, 71-89.

[10] E. Fadell and S. Husseini, Category of loop spaces of open subsets in Euclidean space, Nonlinear Anal. 17 (1991), no. 12, 1153-1161.

[11] F. Giannoni and A. Masiello, On the existence of geodesics on stationary Lorentz manifolds with convex boundary, J. Funct. Anal. 101 (1991), no. 2, 340369.

[12] L. Landau and L. Lifschitz, Théorie des champs, Mir, Moscow, 1970.

[13] A. Masiello, Variational Methods in Lorentzian Geometry, Pitman Research Notes in Mathematics Series, vol. 309, Longman Scientific \& Technical, Harlow, 1994.

[14] E. Mirenghi and M. Tucci, Stationary Lorentz manifolds and vector fields: existence of periodic trajectories, Nonlinear Anal., Ser. A: Theory Methods 50 (2002), no. $6,763-786$.

[15] B. O'Neill, Semi-Riemannian Geometry with Applications to Relativity, Pure and Applied Mathematics, vol. 103, Academic Press, New York, 1983.

[16] R. S. Palais, Morse theory on Hilbert manifolds, Topology 2 (1963), 299-340.

[17] R. K. Sachs and H. Wu, General Relativity for Mathematicians, Springer-Verlag, New York, 1977.

Rossella Bartolo: Dipartimento di Matematica, Politecnico di Bari, Via E. Orabona 4, 70125 Bari, Italy

E-mail address: rosse11a@dm. uniba.it 


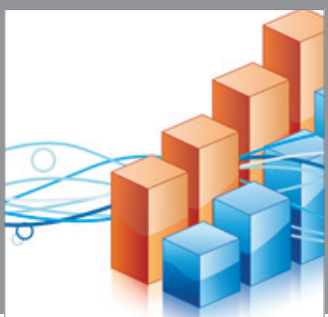

Advances in

Operations Research

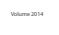

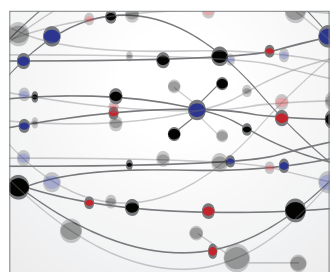

\section{The Scientific} World Journal
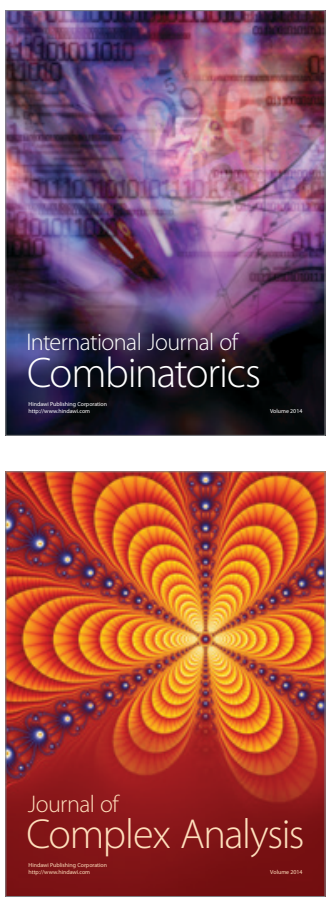

International Journal of

Mathematics and

Mathematical

Sciences
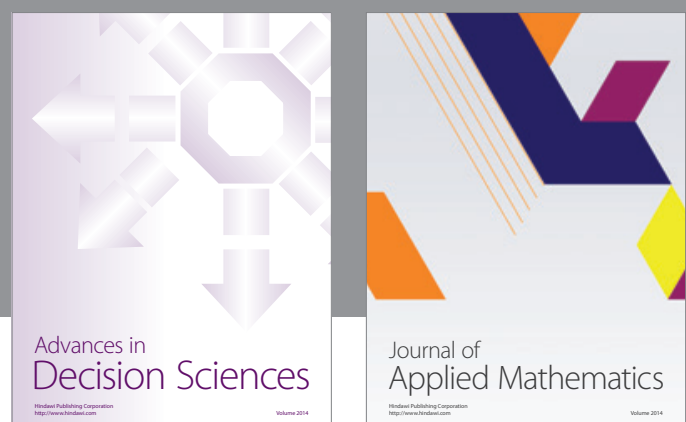

Journal of

Applied Mathematics
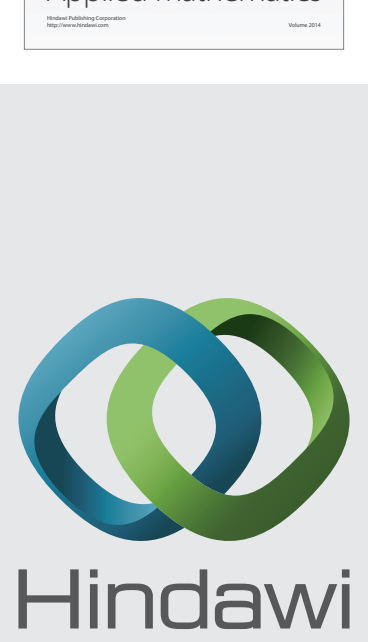

Submit your manuscripts at http://www.hindawi.com
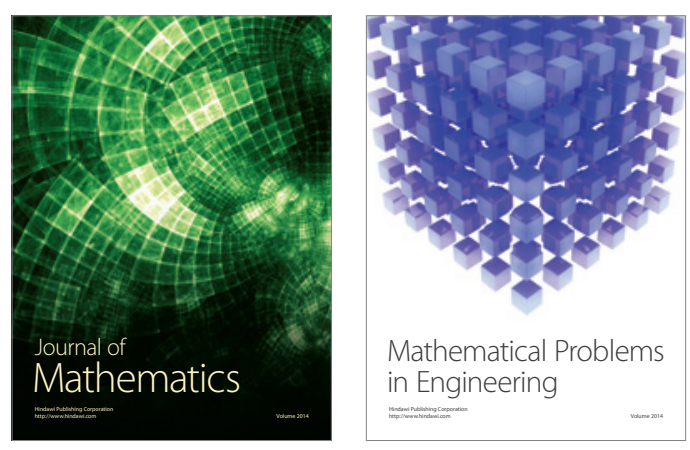

Mathematical Problems in Engineering
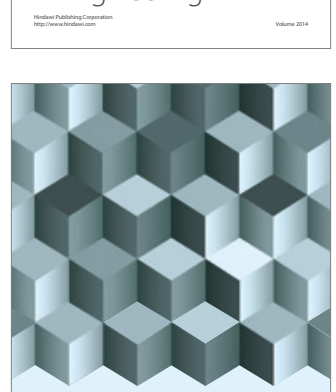

Journal of

Function Spaces
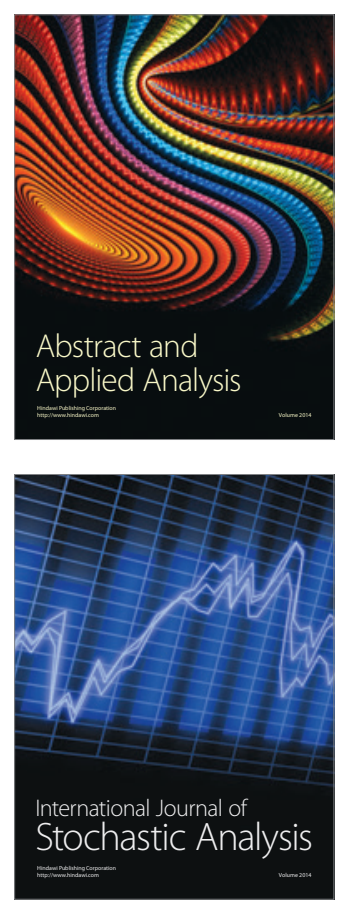

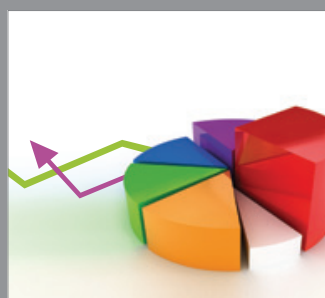

ournal of

Probability and Statistics

Promensencen
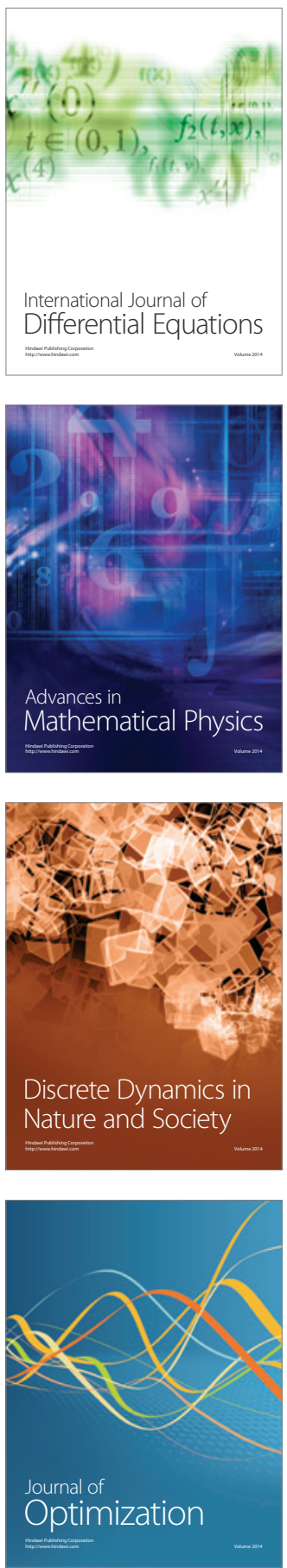